\title{
Effective actions for high energy scattering in QCD and in gravity
}

\author{
L. N. Lipatov ${ }^{1,2, a}$ \\ ${ }^{1}$ Petersburg Nuclear Physics Institute, Gatchina, Orlova Roshcha, 188300, St.Petersburg, Russia \\ ${ }^{2}$ Saint Petersburg State University, St. Petersburg, Russia
}

\begin{abstract}
.
The scattering amplitudes in QCD and gravity at high energies are described in terms of reggeized gluons and gravitons, respectively. In particular, the BFKL Pomeron in $N=4$ SUSY is dual to the reggeized graviton living in the 10-dimensional anti-de-Sitter space. The effective actions for the reggeized gluons and gravitons are local in their rapidities. The Euler-Lagrange equations for these effective theories are constructed and their solutions are used for calculations of corresponding Reggeon vertices and trajectories.
\end{abstract}

\section{Introduction}

The hadron scattering amplitudes $A(s, t)$ at high energies $\sqrt{s}=\sqrt{\left(p_{1}+p_{2}\right)^{2}}$ and fixed momentum transfers $q=\sqrt{-t}$ can be expressed in terms of the $t$-channel partial waves $f_{j}(t)$ having the Regge poles $f_{j}(t) \sim \frac{1}{j-j(t)}$. The Pomeron is a special Regge pole responsible for a slow growth of total cross sections $\sigma_{t}$ and for the fulfilment of the Pomeranchuk theorem on the equality of the particle-particle and particle-anti-particle cross sections at large energies. The exchange of two or more reggeons generates more complicated Mandelstam singularities of $f_{j}(t)$ in the $j$-plane. To take into account all possible Pomeron interactions V.N. Gribov constructed the Reggeon calculus based on the 2+1 quantum field theory of a complex scalar field [1].

The scattering amplitudes in QCD with the gluon quantum numbers in the $t$-channel have the Regge form in the so-called leading logarithmic approximation (LLA) [2]. Here the Pomeron is a colorless composite state of the reggeized gluons. Its wave function in QCD and in other field models with the gauge group $S U\left(N_{c}\right)$ satisfies the BFKL equation [2]. It is remarkable, that the equations for the composite states of several reggeized gluons with the color singlet and octet quantum numbers are integrable at large $N_{c}[3,4]$. Thus, it looks natural to reformulate QCD and other gauge models in terms of the effective degrees of freedom corresponding to the reggeized gluons. Indeed, the gluon Regge trajectory and various reggeon couplings in upper orders of perturbation theory can be calculated from the effective action presented in Ref. [5, 6]. In the $N=4$ SUSY the Pomeron is dual to the reggeized graviton propagating in the anti-de-Sitter space due to AdS/CFT correspondence [79]. The effective action for the high energy scattering in the Einstein gravity was derived in ref. [10]. Below we consider the actions for reggeized gluons and gravitons and the corresponding effective Euler-Lagrange equations.

\footnotetext{
${ }^{\text {a }}$ The investigation is supported by the grants 11.38.223.2015 of SPSU and 14-2200281 of RFBI. e-mail: lipatov@thd.pnpi.spb.ru
} 


\section{Effective theory for high energy processes in QCD}

It is natural to construct the effective theory in QCD for a cluster of quarks, gluons and reggeized gluons having their rapidities $y$ in the interval $\eta$ around its central value $y_{0}$. Apart from the antihermitian matrix $N_{c} \times N_{c}$ corresponding to the gluon field $v_{\mu}^{a}(x)$ we introduce also the fields $A_{ \pm}=$ $p_{1,2}^{\mu} A_{\mu}=A_{0} \pm A_{3}$ describing the production and annihilation of the reggeized gluons

$$
v_{\mu}(x)=-i T^{a} v_{\mu}^{a}(x), A_{ \pm}(x)=-i T^{a} A_{ \pm}^{a}(x),\left[T^{a}, T^{b}\right]=i f^{a b c} T_{c} .
$$

The matrices $T^{a}$ are generators of the gauge group $S U\left(N_{c}\right)$ in the fundamental representation.

Contrary to $v_{\mu}(x)$ the fields $A^{\mp}=A_{ \pm}$are invariant under the local gauge transformations

$$
\delta v_{\mu}=\frac{1}{g}\left[D_{\mu}, \chi(x)\right], D_{\mu}=\partial_{\mu}+g v_{\mu}, \delta A_{ \pm}=0
$$

providing that parameters $\chi(x)$ vanish at $x \rightarrow \infty$. Under the global $S U_{n}$ rotations the Reggeon fields $A^{ \pm}$are transformed as $v_{\mu}$. They satisfy also the kinematical constraints

$$
\partial_{-} A^{-}=\partial_{+} A^{+}=0, \partial_{ \pm}=\frac{\partial}{\partial x^{0}} \pm \frac{\partial}{\partial x^{3}}
$$

corresponding to the fact, that the Sudakov components $\alpha_{i}, \beta_{i}$ of the cluster momenta $k_{i}$ are strongly ordered $\beta_{i} \gg \beta_{i+1}, \alpha_{i} \ll \alpha_{i+1}$ in the multi-Regge kinematics.

The effective action for a cluster of real and virtual particles with their rapidities belonging to the small rapidity interval $\eta$ has the form [5]

$$
S_{e f f}=\int d^{4} x\left(L_{Q C D}+\operatorname{Tr} \partial_{\mu} A^{+} \partial_{\mu} A^{-}\right)+S_{\text {ind }}, S_{\text {ind }}=\operatorname{Tr} \int d^{4} x\left(V_{+} \partial_{\mu}^{2} A^{+}+V_{-} \partial_{\mu}^{2} A^{-}\right)
$$

where $L_{Q C D}$ is the usual QCD lagrangian. The anti-hermitian matrices $V_{ \pm}$in the induced action $S_{\text {ind }}$ are expressed in terms of eikonal amplitudes for a massless particle scattered off the external gluon field $v_{\mu}[5]$

$$
V_{ \pm}=\frac{1}{g} \partial_{ \pm} \frac{1}{D_{ \pm}} \overleftarrow{\partial}_{ \pm} \equiv-\frac{\partial_{ \pm}}{g}+v_{ \pm}-v_{ \pm} \frac{g}{\partial_{ \pm}} v_{ \pm}+v_{ \pm} \frac{g}{\partial_{ \pm}} v_{ \pm} \frac{g}{\partial_{ \pm}} v_{ \pm}+\ldots
$$

where by definition the derivative $\overleftarrow{\partial}$ acts on functions situated to the left from it. The contribution $-\partial_{ \pm} / g$ is negligible in $S_{\text {int }}$, because the fields $A^{ \pm}$do not depend on $x^{ \pm}$. It is natural to define the action of the integral operator $1 / \partial_{ \pm}$in the symmetric form

$$
\frac{1}{\partial_{ \pm}} f\left(x^{ \pm}\right)=\frac{1}{2}\left(\frac{1}{\partial_{0}} \mp \frac{1}{\partial_{3}}\right) f\left(x^{ \pm}\right)=\frac{1}{4}\left(\int_{-\infty}^{x^{ \pm}} d x^{ \pm} f\left(x^{ \pm}\right)-\int_{x^{ \pm}}^{\infty} d x^{\prime \pm} f\left(x^{ \pm \pm}\right)\right) .
$$

The gauge invariance of the action is a consequence of the gauge symmetry of $V_{ \pm}$valid after its integration due to the vanishing of the parameter $\chi(x)$ at $x \rightarrow \infty$.

The matrix $V_{ \pm}$can be written in one of two factorized forms

$$
V_{ \pm}=-\frac{\partial_{ \pm}}{g} O\left(x^{ \pm}\right)=O^{+}\left(x^{ \pm}\right) \frac{\overleftarrow{\partial_{ \pm}}}{g}, O\left(x^{ \pm}\right) \equiv-\frac{1}{D_{ \pm}} \overleftarrow{\partial}_{ \pm}, O^{+}\left(x^{ \pm}\right)=\partial_{ \pm} \frac{1}{D_{ \pm}}
$$

Here the poles $1 / D_{ \pm}$appear from propagators of the massless particles with different rapidities emitting gluons inside the given rapidity interval $\eta$. Their virtualities $\sim k_{ \pm}$are large. Hence it looks 
natural to define the operators $1 / \partial_{ \pm}$with the above principal value prescription but in all terms for the perturbative expansion of $1 / D_{ \pm}$. It would lead to the anti-hermicity of $V_{ \pm}$.

Integrating over $x^{ \pm}$we can present $S_{\text {ind }}$ in terms of asymptotic values of functions $O\left(x^{ \pm}\right)$

$$
S_{\text {ind }}=-\frac{T r}{g} \int d^{2} x_{\perp}\left(\left.\int_{-\infty}^{\infty} d x^{-} O\right|_{x^{+}=-\infty} ^{x^{+}=\infty} \partial_{\sigma}^{2} A^{+}+\left.\int_{-\infty}^{\infty} d x^{+} O\right|_{x^{-}=-\infty} ^{x^{-}=\infty} \partial_{\sigma}^{2} A^{-}\right) .
$$

It is real due to the anti-hermicity of $\left.O\right|_{x^{ \pm}=-\infty} ^{x^{ \pm}=\infty}$.

The product of the operators $O^{+}\left(x^{ \pm}\right)$and $O\left(x^{ \pm}\right)$does not depend on $x^{ \pm}$, but generally $O\left(x^{ \pm}\right)$is not an unitary operator. It is related to our use of the principal value prescription $\theta\left(\left|k^{ \pm}\right|-\epsilon\right) / k^{ \pm}$for propagators $1 / \partial_{ \pm}$. Note, that $\epsilon$ can be chosen here in the form $e^{-\eta}$ where $\eta$ is a low limit for the relative rapidity of neighboring clusters described by the effective action.

One can use the following representation for $1 / D_{ \pm}$in terms of modified $P$-exponents

$$
\frac{1}{D_{ \pm}}=P\left(\frac{e^{-\frac{g}{4} \int_{-\infty}^{x_{ \pm}} d \widetilde{x}^{ \pm} v_{ \pm}}}{e^{-\frac{g}{4} \int_{x_{ \pm}}^{\infty} d \widetilde{x}^{ \pm} v_{ \pm}}}\right) \frac{1}{\partial_{ \pm}} \bar{P}\left(\frac{e^{-\frac{g}{4} \int_{x_{ \pm}}^{\infty} d \widetilde{x}^{ \pm} v_{ \pm}}}{e^{-\frac{g}{4} \int_{-\infty}^{x_{ \pm}} d \widetilde{x}^{ \pm} v_{ \pm}}}\right) .
$$

It allows to write $O\left(x^{ \pm}\right)$in the form

$$
O\left(x^{ \pm}\right)=P\left(\frac{e^{-\frac{g}{4} \int_{-\infty}^{x_{ \pm}} \sqrt{x^{ \pm}} v_{ \pm}}}{e^{-\frac{g}{4} \int_{x_{ \pm}}^{\infty} d \widetilde{x}^{ \pm} v_{ \pm}}}\right) \frac{1}{2}\left(P e^{\frac{g}{4} \int_{-\infty}^{\infty} d \widetilde{x}^{ \pm} v_{ \pm}}+\bar{P} e^{-\frac{g}{4} \int_{-\infty}^{\infty} d \widetilde{x}^{ \pm} v_{ \pm}}\right) .
$$

The first factor here is an unitary matrix and second one is an hermitian operator which does not depend on $x$. We obtain a simple representation for the difference of $O\left(x^{ \pm}\right)$at $x^{ \pm}= \pm \infty$

$$
\left.O\right|_{x^{ \pm}=-\infty} ^{x^{ \pm}=\infty}=\frac{1}{2}\left(P e^{-\frac{g}{4} \int_{-\infty}^{\infty} d \bar{x}^{ \pm} v_{ \pm}}-\bar{P} e^{\frac{g}{4} \int_{-\infty}^{\infty} d \bar{x}^{ \pm} v_{ \pm}}\right)\left(P e^{\frac{g}{4} \int_{-\infty}^{\infty} d \bar{x}^{ \pm} v_{ \pm}}+\bar{P} e^{-\frac{g}{4} \int_{-\infty}^{\infty} d \bar{x}^{ \pm} v_{ \pm}}\right),
$$

which leads to an expression for $S_{\text {ind }}$ having the reality property.

Effective vertices for the reggeized gluon with the indices \pm 1 , color index $c$ and momentum $q$, transformed to $r+1$ gluons with the polarization indices $\mu_{0}, \mu_{1}, \ldots, \mu_{r}$, color indices $a_{0}, a_{1}, \ldots, a_{r}$ and momenta $k_{0}, k_{1}, \ldots, k_{r}$, can be written in the form [5,6]

$$
\Delta_{a_{0} a_{1} \ldots a_{r} c}^{\mu_{0} \mu_{1} \ldots \mu_{r}}=-q^{2} \prod_{i=0}^{r} \delta_{ \pm}^{\mu_{i}} \Delta_{a_{0} a_{1} \ldots a_{r} c}\left(k_{0}^{ \pm}, k_{1}^{ \pm}, \ldots k_{r}^{ \pm}\right), \Delta_{a_{0} c}=\delta_{a_{0} c}, \sum_{i=0}^{r} k_{r}^{ \pm}=q^{ \pm}=0 .
$$

Here $\Delta_{a_{0} \ldots a_{r}}$ has the Bose symmetry and satisfies the recurrent relation (the Ward identity) [5]

$$
\Delta_{a_{0} a_{1} \ldots a_{r}}\left(k_{0}^{ \pm}, k_{1}^{ \pm}, \ldots k_{r}^{ \pm}\right)=\frac{1}{k_{r}^{ \pm}} \sum_{t=0}^{r-1} i f_{a a_{t} a_{r}} \Delta_{a_{0} \ldots a_{t-1} a a_{t+1} \ldots a_{r}}\left(k_{0}^{ \pm}, k_{1}^{ \pm}, \ldots k_{t-1}^{ \pm}, k_{t}^{ \pm}+k_{r}^{ \pm}, k_{t+1}^{ \pm}, \ldots k_{r}^{ \pm}\right),
$$

where $f_{a b c}$ is the structure constant of the gauge group. Thus, these vertices for the principal value prescription do not depend on the color representations of the matrices $v_{ \pm}(x)$ in $S_{\text {ind }}$.

\section{Euler-Lagrange equation for the effective action in QCD}

One can obtain the following expression for the variation of $S_{\text {ind }}$ over $v_{ \pm}$

$$
\delta S_{i n d}=-\operatorname{Tr} \int d^{4} x\left(\delta v_{+} O\left(x^{+}\right) \partial_{\mu}^{2} A^{+} O^{+}\left(x^{+}\right)+\delta v_{-} O\left(x^{-}\right) \partial_{\mu}^{2} A^{-} O^{+}\left(x^{-}\right)\right)
$$


It allows to derive the Euler-Lagrange equations for the effective action in a pure glue-dynamics

$$
\left[D_{\mu}, G^{\mu v}\right]^{\perp}=0,\left[D_{\mu}, G^{\mu \pm}\right]=j_{\text {ind }}^{ \pm}, j_{\text {ind }}^{ \pm}=O\left(x^{ \pm}\right)\left(\partial_{\sigma}^{2} A^{ \pm}\right) O^{+}\left(x^{ \pm}\right) .
$$

Due to the relations $D_{ \pm} O\left(x^{ \pm}\right)=0$ and $\partial_{ \pm} A^{ \pm}=0$ the currents $j_{\text {ind }}^{ \pm}$are conserved

$$
\left[D_{ \pm}, j_{\text {ind }}^{ \pm}\right]=0 .
$$

In the quasi-elastic kinematics, where $A^{+}=0$, in the gauge $v_{-}^{\prime}=0$, we have

$$
j_{\text {ind }}^{\prime-}=\lambda \partial_{\sigma}^{2} A^{-} \lambda^{+}, \lambda=\frac{1}{2}\left(P e^{\frac{g}{4} \int_{-\infty}^{\infty} d \widetilde{x} v_{-}^{\prime}}+\bar{P} e^{-\frac{g}{4} \int_{-\infty}^{\infty} d \widetilde{x} v_{-}^{\prime}}\right)=1
$$

In this case there is an explicit solution of the Euler-Lagrange equations

$$
\widetilde{v}^{\sigma}=\delta_{-}^{\sigma} A^{-}
$$

Thus, the reggeon field $A^{-}$at $A^{+}=0$ has a physical interpretation of the classical solution being a superposition of the shock waves $\delta\left(x^{\mp}-x_{0}^{\mp}\right) \ln \left|x^{\perp}-x_{0}^{\perp}\right|^{2}$.

Inserting the solution of the Euler-Lagrange equation for a general kinematics in $S_{\text {eff }}$, one can obtain a generating function for the reggeized gluon vertices in the tree approximation [5,6]. Note, that even for the quasi-elastic kinematics the Euler-Lagrange equations have other solutions corresponding to more complicated initial and final conditions. The asymptotic behavior of these solutions is fixed at $t=-\infty$ and $t=\infty$ in terms of arbitrary functions $v_{1}(\vec{x})$ and $v_{2}(\vec{x})$, respectively. The effective action calculated on the general solution depending on $A^{ \pm}$and $v_{1,2}(\vec{x})$ gives a possibility to find a generating functional in a tree approximation for all possible gluon and quark transitions on an arbitrary number of reggeized gluons [5]. Furthermore, by calculating the functional integral over the quantum fluctuations $\delta v_{\mu}$ around the classical solutions we can find various effective vertices with loop corrections [11-13].

\section{Effective theory for the high energy gravity}

Due to the AdS/CFT correspondence the $N=4$ super-symmetric gauge theory is equivalent to the 10-dimensional super-string model living on the 10-dimensional anti-de-Sitter space [7-9]. Here the BFKL Pomeron is dual to the reggeized graviton (see, for example, [14]). Note, that the graviton Regge trajectory and its various couplings in a leading order are known [15].

The high energy effective action in the Einstein gravity is constructed for a cluster of gravitons and reggeized gravitons having the rapidities in an interval around their central value [10]. Apart from the usual Einstein-Hilbert action and a kinetic term for the reggeon fields $A^{ \pm \pm}$

$$
S=-\frac{1}{2 \kappa^{2}} \int d^{4} x\left(\sqrt{-g} R+\partial_{\sigma} A^{++} \partial_{\sigma} A^{--}\right)+S_{\text {ind }},
$$

it contains the additional contribution $S_{\text {ind }}$

$$
S_{\text {ind }}=-\frac{1}{2 \kappa^{2}} \int d^{4} x\left(\frac{j_{++}}{2} \partial_{\sigma}^{2} A^{++}+\frac{j_{--}}{2} \partial_{\sigma}^{2} A^{--}\right),
$$

where the induced currents $j_{ \pm \pm}$are functionals of the metric tensor $g^{\mu \nu}$. 
The fields $A^{ \pm \pm}$describe the production and annihilation of reggeized gravitons in the $t$-channel. They are invariant under the general coordinate transformations reduced to the Poincare subgroup at large distances and satisfy the kinematical constraints

$$
\partial_{+} A^{++}=\partial_{-} A^{--}=0,
$$

corresponding to the strong ordering of the Sudakov components for momenta of produced clusters in the multi-Regge kinematics. The graviton fields $h_{\mu \nu}$ are introduced as fluctuations of $g_{\mu \nu}$ around the Minkowsky metric tensor $\eta_{\mu v}$ having the diagonal structure $(1,-1,-1,-1)$.

The functional form of the current $j_{ \pm \pm} \equiv \partial_{ \pm} j^{\mp}$ is fixed by the general covariance of the action. It turns out, that the function $\omega^{\mp}=j^{\mp}+2 x^{\mp}$ satisfies the Hamilton-Jacobi equation [10]

$$
g^{\rho \sigma}\left(\partial_{\rho} \omega^{\mp}\right)\left(\partial_{\sigma} \omega^{\mp}\right)=0 .
$$

Physically this equation describes the light-front wave moving in an arbitrary gravitational field. On the other hand, the quantity $x^{\prime \mp}=\omega^{\mp} / 2$ can be considered as a light-cone component of the coordinate transformation $x^{\prime}(x)$ to the system with the global light-cone time $x^{\prime F}$, corresponding to the equality $g^{\prime \mp \mp}=0$. The Hamilton-Jacobi equation does not fix the global light-cone time system completely. It is naturally to impose on $g^{\prime \rho \sigma}$ more restrictive constraints

$$
g^{\rho \mp}=\eta^{\rho \mp}, \eta^{\mp \mp}=\eta_{\perp}^{\rho \mp}=0, \eta^{ \pm \mp}=1 .
$$

In this inertial system we have in particular $g^{\prime \rho \mp} \frac{\partial}{\partial x^{\prime \rho}}=\frac{\partial}{\partial x^{\prime \pm}}$.

Note, that the induced action can be expressed only in terms of the functions $\omega^{\mp}$ at $x^{ \pm}=\infty$

$$
S_{\text {ind }}=\frac{1}{2 \kappa^{2}} \int d^{2} x^{\perp}\left(\left.\int_{-\infty}^{\infty} \frac{d x^{-}}{4} \omega^{-} \partial_{\sigma \perp}^{2} A^{++}\right|_{x^{+}=-\infty} ^{x^{+}=\infty}+\left.\int_{-\infty}^{\infty} \frac{d x^{+}}{4} \omega^{+} \partial_{\sigma \perp}^{2} A^{--}\right|_{x^{-}=-\infty} ^{x^{-}=\infty}\right) .
$$

\section{Classical equations for the effective gravity}

The variations $\delta g^{\mu \nu}$ and $\delta \omega^{\mp}$ are not independent due to the Hamilton-Jacobi equation for $\omega^{\mp}$

$$
\partial_{\mu} \omega^{\mp} \partial_{\nu} \omega^{\mp} \delta_{\tau} g^{\mu \nu}+2 g^{\rho \sigma} \partial_{\rho} \omega^{\mp} \delta_{\tau} \partial_{\sigma} \omega^{\mp}=0
$$

and expressed through the corresponding infinitesimal shifts in the proper time $\tau$

$$
\delta_{\tau} g^{\mu \nu}=d \tau\left(\partial_{\sigma} g^{\mu \nu}\right) \frac{d}{d \tau} x^{\sigma}, \delta_{\tau} \partial_{\sigma} \omega^{\mp}=d \tau \frac{d}{d \tau} \partial_{\sigma} \omega^{\mp} .
$$

They can be calculated with the help of the Hamilton equations

$$
\frac{d}{d \tau} x^{\sigma}=g^{\sigma \rho} \partial_{\rho} \omega^{\mp}, \frac{d}{d \tau} \partial_{\sigma} \omega^{\mp}=-\frac{1}{2}\left(\partial_{\sigma} g^{\mu \nu}\right) \partial_{\mu} \omega^{\mp} \partial_{\nu} \omega^{\mp} .
$$

Because $x^{\prime \mp}=\omega^{\mp} / 2$ are light-cone components of $x^{\prime}(x)$ in systems with $g^{\prime \mp \mp}=0$ we have

$$
2 g^{\rho \sigma} \partial_{\rho} \omega^{\mp} \delta_{\tau} \partial_{\sigma} \omega^{\mp}=-2 d \tau g^{\prime \mp \mp} \frac{\partial g^{\mu \nu}}{\partial x^{\prime \chi}} \partial_{\mu} \omega^{\mp} \partial_{\nu} \omega^{\mp}=-2 d \tau \frac{\partial g^{\mu \nu}}{\partial x^{\prime \pm}} \partial_{\mu} \omega^{\mp} \partial_{\nu} \omega^{\mp}=4 \delta_{\tau} \frac{\partial \omega^{\mp}}{\partial x^{\prime \pm}}
$$

where the property $g^{\prime \mp}=\eta^{{ }^{\mp}}$ of the global light-cone time inertial systems was used. By integrating these equalities over the coordinates $x^{\prime}$ it is possible to verify the relations

$$
\int d^{4} x^{\prime} 4 \delta_{\tau} \frac{\partial \omega^{\mp}}{\partial x^{\prime \pm}} \partial_{\sigma \perp}^{2} A^{ \pm \pm}\left(x^{\prime}\right)=\int d^{4} x \sqrt{-g} 2 g^{\rho \sigma} \partial_{\rho} \omega^{\mp} \delta_{\tau}\left(\partial_{\sigma} \omega^{\mp}\right) \partial_{\sigma \perp}^{2} A^{ \pm \pm}\left(x^{\prime}\right),
$$


where we performed a transition to the integration over the coordinates $x$ with the general metric tensor $g^{\mu \nu}$ by introducing the additional factor $\sqrt{-g}$ for the covariance of this expression.

The left hand side of last equality after its integration over $x^{\prime \pm}$ enters in the variation of the induced action

$$
\delta S_{\text {ind }}=\frac{1}{2 \kappa^{2}} \int d^{2} x^{\perp}\left(\left.\int_{-\infty}^{\infty} \frac{d x^{-}}{4} \delta \omega^{-} \partial_{\sigma \perp}^{2} A^{++}\right|_{x^{+}=-\infty} ^{x^{+}=\infty}+\left.\int_{-\infty}^{\infty} \frac{d x^{+}}{4} \delta \omega^{+} \partial_{\sigma \perp}^{2} A^{--}\right|_{x^{-}=-\infty} ^{x^{-}=\infty}\right) .
$$

Therefore, taking into consideration again the above relation between $\delta_{\tau} g^{\mu \nu}$ and $\delta_{\tau} \omega^{\mp}$ on particle trajectories, one can present the variation of $S_{\text {ind }}$ over $g^{\mu v}$ in a simple covariant form

$$
\delta S_{\text {ind }}=-\frac{1}{2 \kappa^{2}} \int d^{4} x \sqrt{-g} \delta g^{\mu \nu} \frac{1}{4}\left(\partial_{\mu} \omega^{-} \partial_{\nu} \omega^{-} \partial_{\sigma \perp}^{2} A^{++}\left(x^{\prime}\right)+\partial_{\mu} \omega^{+} \partial_{\nu} \omega^{+} \partial_{\sigma \perp}^{2} A^{--}\left(x^{\prime}\right)\right) .
$$

We remind, that the factor $\sqrt{-g}$ is obtained as a result of the transformation from a special coordinate system with the global light-cone time $x^{\prime \mp}$ to a general system with coordinates $x$.

The above expression for $\delta S_{\text {ind }}$ allows one to calculate easily the induced energy-momentum tensor $\theta_{\mu \nu}$ in the Euler-Lagrange equations

$$
R_{\mu \nu}-\frac{1}{2} g_{\mu \nu} R=-\theta_{\mu \nu}, \theta_{\mu \nu}=\partial_{\mu} x^{\prime-} \partial_{\nu} x^{-} \partial_{\sigma}^{2} A^{++}\left(x^{\prime}\right)+\partial_{\mu} x^{\prime+} \partial_{\nu} x^{\prime+} \partial_{\sigma}^{2} A^{--}\left(x^{\prime}\right),
$$

where $x^{\prime \pm}=\omega^{ \pm} / 2$ are coordinates in the corresponding global light-cone systems. Below this result is obtained independently from considerations related to the general covariance of the Euler-Lagrange equations.

To begin with, in a quasi-elastic kinematics, where $A^{++}=0$, it is convenient to work in the inertial system with the metric tensor obeying the constraints $g^{\prime \rho+}=\eta^{\rho+}$ with the global light-cone time $x^{\prime+}$, because here the energy-momentum tensor is $T_{\mu \nu} \sim \delta_{\mu}^{+} \delta_{v}^{+}$and there is a simple classical solution

$$
g^{\prime \rho \sigma}=\eta^{\rho \sigma}+\delta_{-}^{\rho} \delta_{-}^{\sigma} A^{--}\left(x^{\prime}\right) .
$$

It is a superposition of the plane-wave solutions of Aichelburg and Sexl with the gravitation centers situated at $x^{+}=z^{+}, x^{\perp}=z^{\perp}$ and distributed with the weight function $\partial_{\tau \perp}^{2} A^{--}\left(z^{+}, z^{\perp}\right)$. The coordinate transformation $x^{\prime}=x^{\prime}(x)$ to the light-cone time system satisfies the equations

$$
g^{\mu \nu} \frac{\partial x^{\prime \rho}}{\partial x^{\mu}} \frac{\partial x^{\prime+}}{\partial x^{\nu}}=\eta^{\rho+}
$$

and the tensors $\theta_{\mu \nu}$ and $T_{\rho \sigma}$ in these two systems are related as follow

$$
\theta_{\mu \nu} \frac{\partial x^{\mu}}{\partial x^{\prime \rho}} \frac{\partial x^{\nu}}{\partial x^{\prime \sigma}}=T_{\rho \sigma} .
$$

It means, that the covariant energy-momentum tensor for the quasi-elastic kinematics is

$$
\theta_{\mu \nu}=\partial_{\mu} x^{+} \partial_{\nu} x^{\prime+} \partial_{\sigma}^{2} A^{--}\left(x^{\prime}\right),
$$

where partial derivatives $\partial_{\mu} x^{\prime+}$ are found from the solution of the Hamilton-Jacobi equation

$$
g^{\mu v} \partial_{\mu} x^{++} \partial_{v} x^{++}=0
$$

The covariant energy-momentum tensor for a general kinematics where $A^{ \pm \pm} \neq 0$ is conserved

$$
D_{\mu} \theta^{\mu v}=0
$$


due to the kinematical constraints $\partial_{ \pm} A^{ \pm \pm}=0$. As a consequence of the Hamilton-Jacobi equation for $x^{\prime \pm}$ the tensor $\theta_{\mu \nu}$ is traceless

$$
g^{\mu v} \theta_{\mu v}=0 .
$$

Thus, we constructed the generally covariant Euler-Lagrange equations for the effective action in the high energy gravity. In a quasi-elastic kinematics with $A^{++}=0$ one of their classical solutions at the coordinate system with the global light-cone time $x^{\prime+}$ has a simple form $g^{\prime--}=A^{--}$. In a general quasi-multi-Regge kinematics there are other solutions parameterized by the functions with positive and negative frequencies at $t \rightarrow-\infty$ and $t \rightarrow \infty$, respectively. The effective action calculated on these solutions allows to construct a generating functional for the effective multi-graviton scattering amplitudes with an arbitrary number of reggeized gravitons in a tree approximation and to reproduce independently the known results for the graviton Regge trajectory and effective vertices [15]. The integration over fluctuations around the classical solutions gives a possibility to calculate loop corrections to reggeized graviton interactions and to reggeon vertices. Note, that using the one-loop Regge trajectory obtained in Refs $[10,15]$, the graviton scattering amplitude in the double-logarithmic approximation was constructed in [16]. A possible generalization of our approach to the super-gravity in the 10-dimensional AdS space will be interesting for the construction of the Gribov Pomeron calculus in $\mathrm{N}=4$ SUSY.

\section{References}

[1] V. N. Gribov, Sov. Phys. JETP 26, 414 (1968).

[2] L. N. Lipatov, Sov. J. Nucl. Phys. 23, 338 (1976);

V. S. Fadin, E. A. Kuraev, L. N. Lipatov, Phys. Lett. B 60, 50 (1975);

E. A. Kuraev, L. N. Lipatov, V. S. Fadin, Sov. Phys. JETP 44, 443 (1976);

Ya. Ya. Balitsky, L. N. Lipatov, Sov. J. Nucl. Phys. 28, 822 (1978).

[3] L. N. Lipatov High energy asymptotics of multi-colour QCD and exactly solvable lattice models, hep-th/9311037, unpublished.

[4] L. N. Lipatov, J. Phys. A 42:304020 (2009).

[5] L. N. Lipatov, Nucl. Phys. B 452, 369 (1995); Phys. Rept. 286, 131 (1997).

[6] E. N. Antonov, L. N. Lipatov, E. A. Kuraev, I. O. Cherednikov, Nucl. Phys. B 721, 111 (2005).

[7] J. M. Maldacena, Adv. Theor. Math. Phys. 2, 231 (1998).

[8] S. S. Gubser, I. R. Klebanov, A. M. Polyakov, Phys. Lett. B 428, 105 (1998).

[9] E. Witten, Adv. Theor. Math. Phys. 2, 253 (1998).

[10] L. N. Lipatov, Phys. Part. Nucl. 44391 (2013), arXiv: 1105.31277 [hep-ph].

[11] V. S. Fadin, L. N. Lipatov, Phys. Lett. B 429, 127 (1998);

M. Ciafaloni and G. Camici, Phys. Lett. B 430, 349 (1998).

[12] A. V. Kotikov, L. N. Lipatov, Nucl. Phys. B 582, 19 (2000).

[13] A. V. Kotikov, L. N. Lipatov, Nucl. Phys. B 661, 19 (2003).

[14] A. V. Kotikov, L. N. Lipatov, Nucl. Phys. B 874, 889 (2013).

[15] L. N. Lipatov, Phys. Lett. 116, 411 (1982).

[16] J. Bartels, L. N. Lipatov, A. Sabio Vera, J. High Energy Phys. 07056 (2014). 